\title{
Light, water and physics in Monet painting
}

\author{
Pierre Coullet ${ }^{1}$ and Yves Pomeau ${ }^{2}$ \\ ${ }^{1}$ Université de Nice-Sophia Antipolis, Nice, France \\ ${ }^{2}$ Ladhyx, Ecole Polytechnique, Palaiseau, France
}

\begin{abstract}
A common observation is the one of reflection of the Sun or the Moon by the slightly perturbed surface of water. In "Impression, soleil levant", now in Marmottant museum in Paris, Monet painted the luminous stripe resulting of this reflection when the source is low on the horizon. As we explain this stripe originates from the fluctuations of the angle of the reflecting surface when they are big enough to spread the multiple images to make them overlap, which requires that the fluctuations of the surface angle are of the same order as the angle of the Sun (or Moon) above the horizon. At higher angle the stripe become a set of non overlapping points representing each the reflected image of the source. This makes an interesting percolation transition by a continuous change of a parameter.
\end{abstract}

Man has forever observed the ocean and the reflection of light by it. In the city of Nice, a morning the authors had a chance to, admire the glitter of the sea, obviously due to the specular reflection of the sun by a slightly agitated surface of the sea, with likely an elevation of this surface that is random in space and time. This blinking has drawn the attention of physical oceanography with the idea that one could derive from its analysis statistical properties of the wave pattern. As far we know, this remains a challenging problem because of the large number of parameters involved. We shall not dwell into this compiex problem and limit ourselves to simple observations which do not seem to have been made before.

Shortly after WWII Munk and collaborators tried to find a way to measure the state of the wave system in the Pacific ocean, a reasonable attempt because this Ocean is so big that there is no way to have a dense enough set of buyos to monitor accurately the wave system in this big Ocean. Various theories were proposed in relation with this line of research, but it does not seem that they take into account the phenomenon painted by Monet in "Impression, soleil levant" that we describe below (see Figure 1). Piotr Szymczak pointed out to us a small book devoted to the Wonders of physics [2] and specifically its chapter 5 where the phenomenon examined below is considered, namely the existence of bands of light created by the reflection of either the Sun or the moon on a random surface at small incidence. This chapter 5 gives no detailed explanation however, and only says that such a stripe is more frequent at small incidence without explaining why. We can only recommend this excellent book, full of new perspectives and of good remarks.

To come to the great painter in the title of this article, Claude Monet, he painted very accurately the reflected image of the Sun on the water in the harbor of Le Havre, shortly after Sunrise. He noticed that, even if the reflecting surface is almost planar the reflected image is not a disc as it would be for a perfect plane mirror, but instead a stripe approximately of the same width as the apparent radius of the Sun, a stripe oriented in the direction of the Sun and seeming to go to infinity.

Cézanne said of Monet: he is an eye only but what an eye!. Monet did not paint his "Impression, soleil levant" on the spot, this pa!nting being particularly complex in its organization and in the display of different colors .Most likely he had to paint it in his workshop by relying on his fantastic visual memory. This memory showed that the image of the Sun is not the result of a simple reflection on the sea surface but tha this one played so to speak an active role in diluting the image into a luminous stripe of warm colors. This transformation by the surface fluctuations from a geometric reflected image into a stripe occurs at low incidence only. As we shall explain at higher incidences the image becomes a set of disconnected points of light. This will be discussed via order of magnitude estimates, all relying on the law of geometrical optics for reflection, a law going back to the ancient Greeks. Our analysis will show, hopefully, that order can be put in a complex situation without dwelling into an analysis with the paraphernalia of a formal statistical approach. This emphasizes also the generic character of our results, partly because of the simplicity of their derivation.

One thinks quite naturally to two parameters to characterize the fluctuations of the sea surface: the range of those fluctuations along the surface, which can be see also as the average wavelength of the waves making those fluctuations. We shall assume that the height is of order of magnitude as this wavelength multiplied by a small number of order 0.15. This small number gives the order of magnitude of the slope of the waves to be compared to two other parameters, the apparent diameter of the Sun, denoted as $\alpha$ and the angle of the Sun with respect to the horizontal.

A theory of the phenomenon we wish to study begins with the Cartesian equation of the reflecting surface written in its simplest form as

$$
z=h(x, y)
$$

, $z$ being the elevation of the surface and $(x, y)$ the coordinates in the horizontal plane. We assume furthermore that 


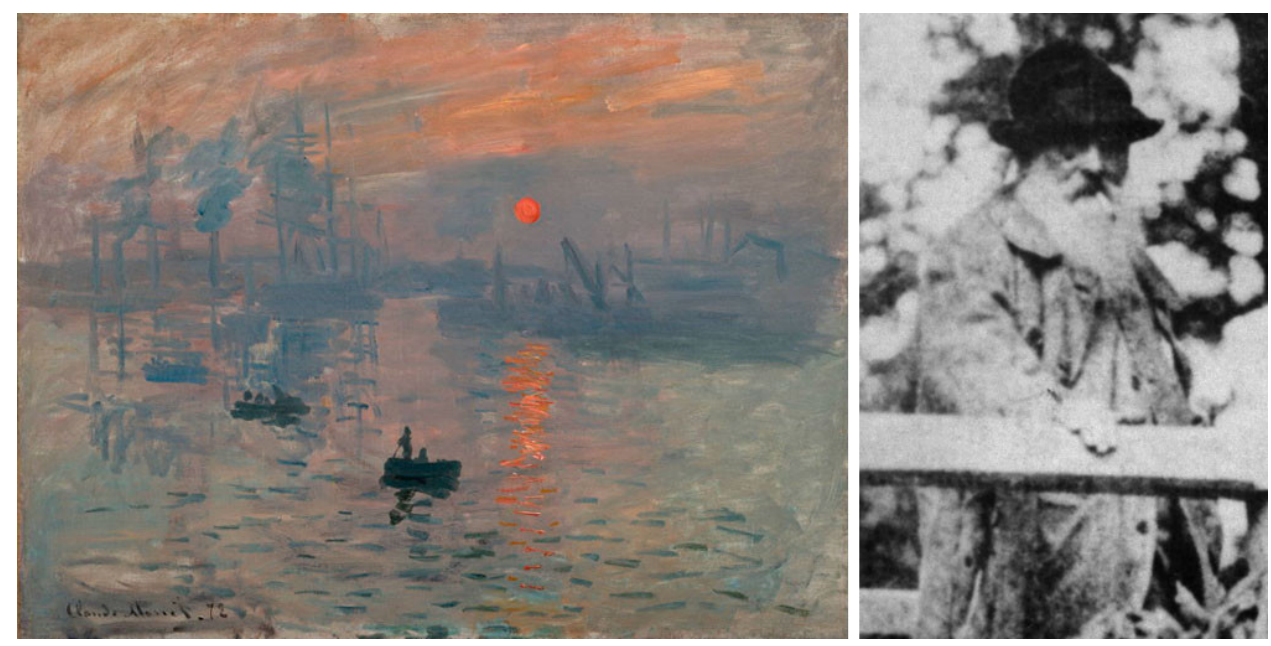

FIG. 1: Impression, soleil levant, Monet (1872)

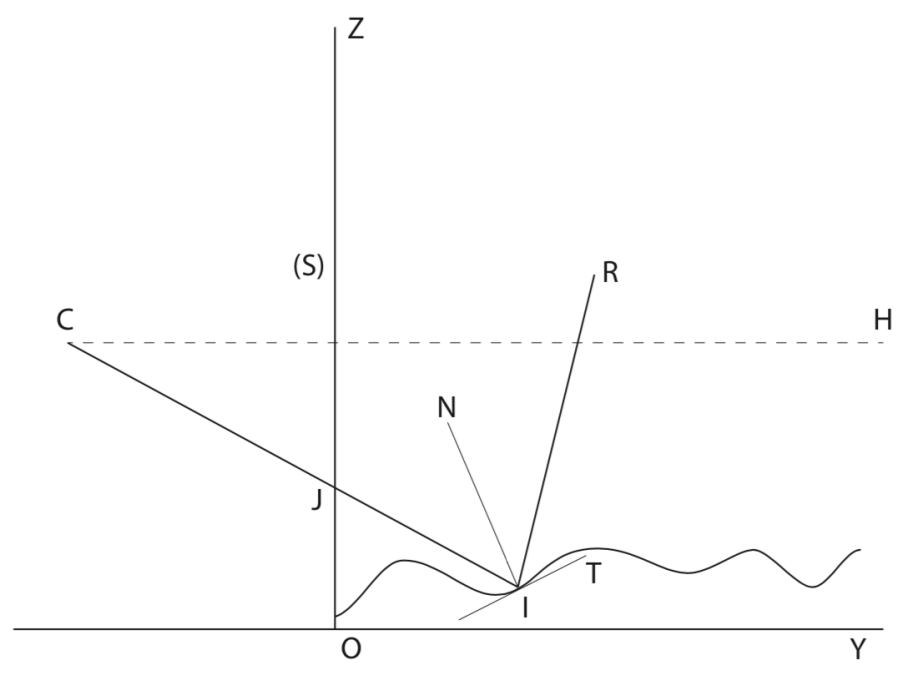

FIG. 2: This figure is to explain the ray tracing algorithm used for numerical simulations. A cross section is displayed, such as the sea surface is in the plane $z=0$. It crosses the shore on the line $y=z=0$. A screen is put in the plane $y=0$. From a point $C$ a ray is sent that crosses the reflecting sea surface at $I$. One compute then the direction of the reflected ray in the solid angle of the Sun seen from $I$, one points a point $J$ of a different color than the background. In the simulation to get rid of effects of discrete representation of the surface the sea is represented by a set of tiny mirrors whose normal are taken randomly between two values.

the reflecting surface is only slightly tilted with respect to the horizontal. Therefore the gradients of $h(x, y)$ are small. This permits to simplify the equations for the reflected ray, given that the incident ray is nearly horizontal.

Figure 2 explains how to relate the condition of specular reflection to the position of the observer (Monet sitting near the quay) to the instantaneous shape of the reflecting surface. Two conditions have to be written for a ray incident along the direction indexed by the unit vector $\mathbf{n}$, the direction of the reflect ray being along the unit vector $\mathbf{m}$. The relation between $\mathbf{n}$ and $\mathbf{m}$ depends also on the unit vector $\mathbf{N}$ normal to the reelecting surface. It reads:

$$
\mathbf{n}=\mathbf{m}-2 \mathbf{N}(\mathbf{N} \cdot \mathbf{m}) .
$$

In the limit of a small slope of the surface the Cartesian components of $\mathbf{N}$ are $\left(-h_{, y}, h_{, x}, 1\right)$ where both $h_{, x}=\frac{\partial h}{\partial x}$ and 
$h_{, y}=\frac{\partial h}{\partial y}$ are small. Whence the two components of $\mathbf{n}$ and $\mathbf{m}:$

$$
h_{, y}=\frac{m_{x}-n_{x}}{2 m_{z}}
$$

and

$$
h_{, x}=\frac{n_{y}-m_{y}}{2 m_{z}}
$$

For two given unit vectors $\mathbf{m}$ and $\mathbf{n}$, and given equation $z=h(x, y)$, the conditions above yield a discrete set of solutions that are pairs of values of $x$ and $y$. This explains the glitter observed when the Sun is well above the horizon. In this case each of the points where a deflection takes place that sends the reflected ray toward the observer yiedls an apparent luminous point on the surface. In this case the angular diameter of the Sun is too small to have overlapping images. The glitter is due to the fact that, as the surface changes in the course of time, the points where the reflections takes place change and give the impression of appearing and disappearing all the time. To estimate their apparent lifetime one can reason as follows. Let $\lambda$ be the range of correlation of the height of the waves, practically their wavelength. Along such a length the slope of the surface will change by an angle $\delta \theta$, an angle that we shall assume to be larger than the angular diameter of the Sun (or of the Moon which is nearly the same). By definition the time $\tau$ during which the ray will continue to reach the observer is such that during this time the change of slope in the equation for the point $(x, y)$ is compensated by the choice of another point $\mathrm{n}$ the angular diameter of the Sun to define the direction of the incoming ray. If $\delta \theta$ is much bigger than the apparent angle, the compensation by the change of point on the disc of the Sun will be possible only on a period of time much shorter than the period of the wave. We assume more precisely that this time $\tau$ is the time necessary for the slope of the wave to change by an amount of the order of the angular diameter of the Sun. This period s related to the wavelength by Newton's relation and has the order of magnitude $\left(\frac{\lambda}{g}\right)^{1 / 2}$, with $g$ acceleration of gravity. During this time the slope of the surface changes by $\delta \theta$, larger than $\alpha$ for a established sea state. More specifically one can assume that this time is obtained by multiplying the time $\tau$ by the small ratio of the angular diameter $\alpha$ to the total variation of the slope. This yields the following estimate of the coherence time of the fluctuations:

$$
t_{c o r r}=\frac{\alpha}{\delta \theta}\left(\frac{\lambda}{g}\right)^{1 / 2} .
$$

Let us remind the reader that this concerns the blinking of isolated points. Nevertheless this is also the order of magnitude of the correlate time typical of fluctuations of the reflected light when the image of theSun is more or less continuous as in Monet painting, a continuity following the overlap of images of the solar disc by the sea surface, the problem we shall consider now.

We shall derive from the formal equations giving the location of the point of reflection an understanding off what is observed by using the various parameters involved in the problem, parameters which are predominantly angles: local angle defining the slope of the surface and angular diameter of the Sun. Let $\theta$ be the angle of the Sun with respect to the horizon and recall that $\lambda$ denotes the range of correlations of the fluctuations of the reflecting surface, and that $\delta \theta$ is the order of magnitude of the angle of deviation of the normal $\mathbf{N}$ with respect $t$ the vertical. Inside a piece of the surface of area $\lambda^{2}$ all orientations of the normal are represented inside a small closed curve on the unit sphere that we assimilate to a small circle of radius $\delta \theta$. Another relevant parameter is the ratio $\frac{\alpha}{\delta \theta}$ assumed to be small. Lastly let $l \approx \frac{d}{\theta}$ be the horizontal distance between the observer and the average point of reflection. Let $\delta l_{L} \ll l$ be the fluctuation of $l$ due to the change of local orientation of the surface, a fluctuation assumed to be small with respect to $\theta$. A small change in the relationship between $l$ and $\theta$ gives:

$$
\delta l_{L} \sim \frac{\delta \theta d}{\theta^{2}}
$$

that is valid $\mathrm{n}$ the longitudinal direction along the reflected ray. In the perpendicular direction the change of position of the point of refection due to the change of orientation of the reflecting surface is

$$
\delta l_{T} \sim \delta \theta d
$$

In order to have many reflected rays reaching the observer, $\delta l_{L}$ must be bigger than the correlation length of the fluctuations of the surface, that is $\delta l_{L} \gg \lambda$ of the same order of magnitude as $\lambda \ll \frac{\delta \theta d}{\theta^{2}}$. 
If this condition is satisfied there is about one ray reaching the observer coming from each area of correlation in region distant of the point of specular reflection less than $\delta l_{L}:$. AT smaller distances one finds a point of reflection of an angle of deviation less than the fluctuation of the slope of the surface. .

Rays reflected at infinity could reach the observer $f$ the fluctuation $\delta \theta$ is of the order of magnitude or bigger than $\theta$, because in this case the lccal variation of the angle allows to reach the point of observation at infinity with a brazing incidence, this assuming a flat sea surface, the curvature of the Earth could change a bit this picture, infinity becoming then the point of tangency of straight lines issued from the observer and tangent to the Earth surface.

Let us consider now the effect of angular width $\alpha$ of he source. If this angles very small, the image seen by the observer will be a set of points, all issued from the same area of correlation of slope of the surface of the sea. If $n$ the contrary $\alpha$ is big enough the reflected image will fill continuously the field by overlapping of all the point images. Therefore it is natural to compare $\alpha$ et $\delta \theta$.

If $\frac{\alpha d}{\theta^{2}} \ll \lambda$, the images originating from the various area of correlation will be disconnected and the impression will she one $\mathrm{f}$ a set of independent points.

On the contrary many points of reflection will exist in the opposite limit $\frac{\delta \theta d}{\theta^{2}} \gg \lambda$. The existence of apparent disconnected points of reflection implies $\alpha \ll \theta$. Let us assume that the opposite is true, namely that

$$
\lambda \ll \frac{\alpha d}{\theta^{2}},
$$

and that

$$
\lambda \ll \frac{\delta \theta d}{\theta^{2}}
$$

In this case one has an image with an apparent continuous stripe by overlapping of images of the Sun (or the Moon). This happens if there are many points of reflection inside an area $\mathrm{f}$ correlation. This area s of order of magnitude $\delta l_{L} \delta l_{T}$. Therefore th image of a continuous stripe impies $\delta l_{L} \delta l_{T} \gg \lambda^{2}$. In this case there is about one reflected ray reaching the observer issuing from each area of correlation. This image has the same angular width as the source because it is made of rays spread along the axis source-observer.

When the angle $\theta$ increases one observes a transition of the percolation type in the reflected image. This transition occurs in the longitudinal direction, as watt happens in the transverse direction is barely dependent on the angle $\theta$.

In the continuous stripe seen at low incidences there is also some time dependence of the intensity of reflected light, less visible than the one of isolated points at higher incidences. This time dependence is a varitation of the intensity around an average value, whereas at higher incidences one sees blinking of point-like sources appearing and diapering at random locations. As we have seen the typical time for the blinking is $\left(\frac{2 \pi \lambda}{g}\right)^{1 / 2}$ for gravity waves. It could be that part of the blinking is due to capillary waves of shorter wavelength than the gravity waves. For water the transition occurs at a fraction of a centimeter. This transition (particularly through its effect on the time correlation of the intensity of blinking) is of interest. The figures 3 and 4 display two examples of continuous luminous stripe seen by the authors just after sunrise on the mediterranean shore of Southern France.

Figures 5 and 6 display the result of a numerical simulation of the transition from a continuous stripe low incidence (figure 5) to a set of blinking point at higher incidences (figure 6). The two figures use an algorithm of ray tracing based on the idea of representing the sea by a cut $(x, z), x$ the horizontal coordinate and $z$ vertical. Without fluctuation the sea surface is the plane $z=0$ The shore is the line $y=z=0$. 


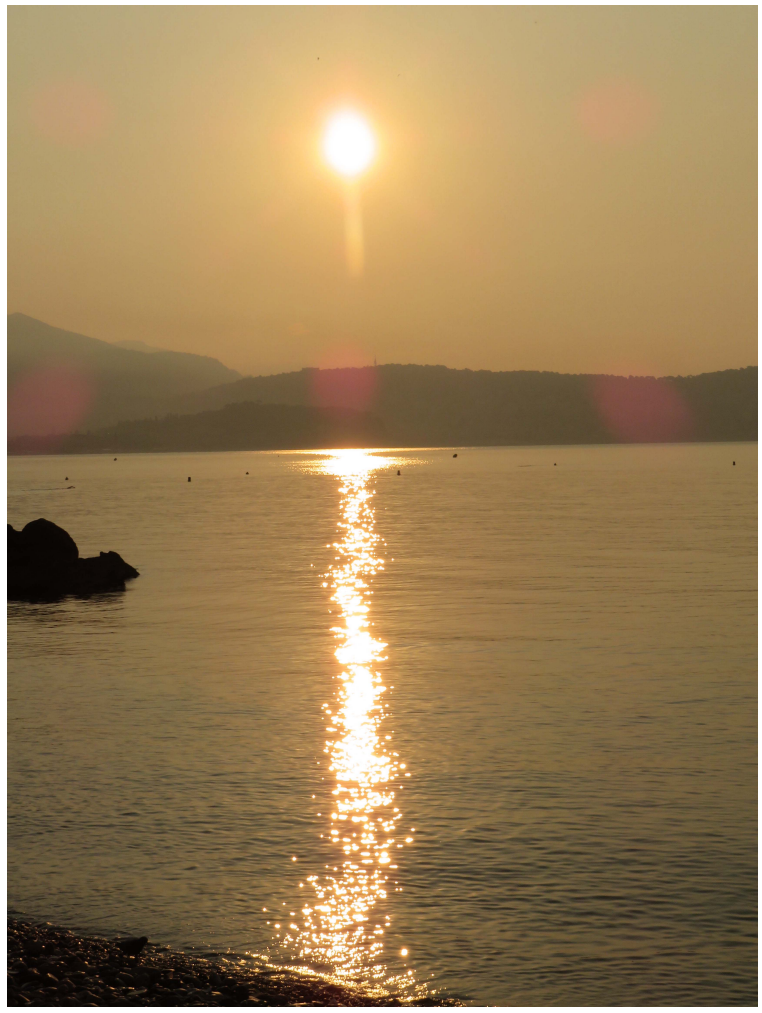

FIG. 3: Sunrise on the Mediterranean in Nice

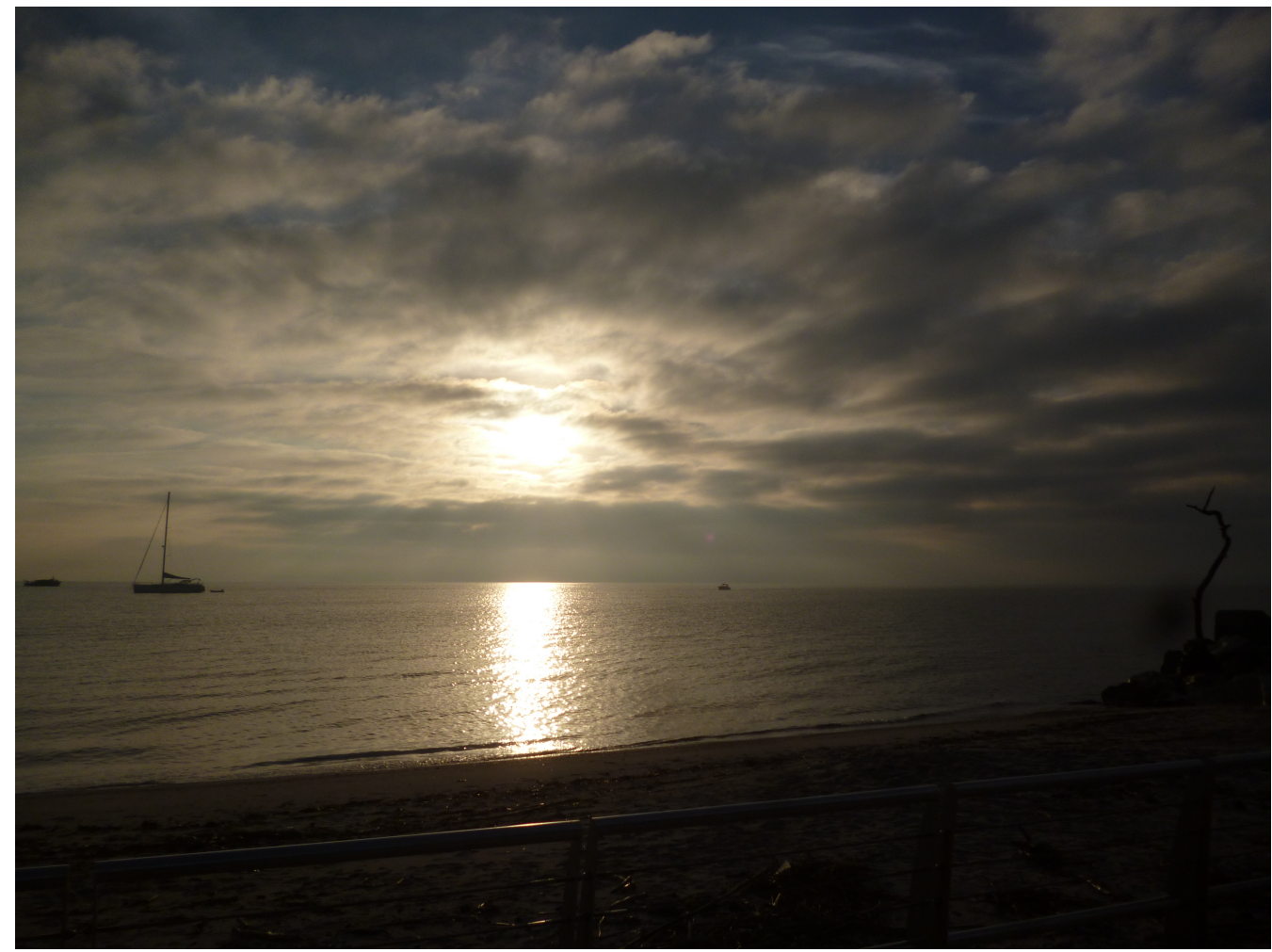

FIG. 4: Early morning view taken east of the Giens peninsula in Southern France 


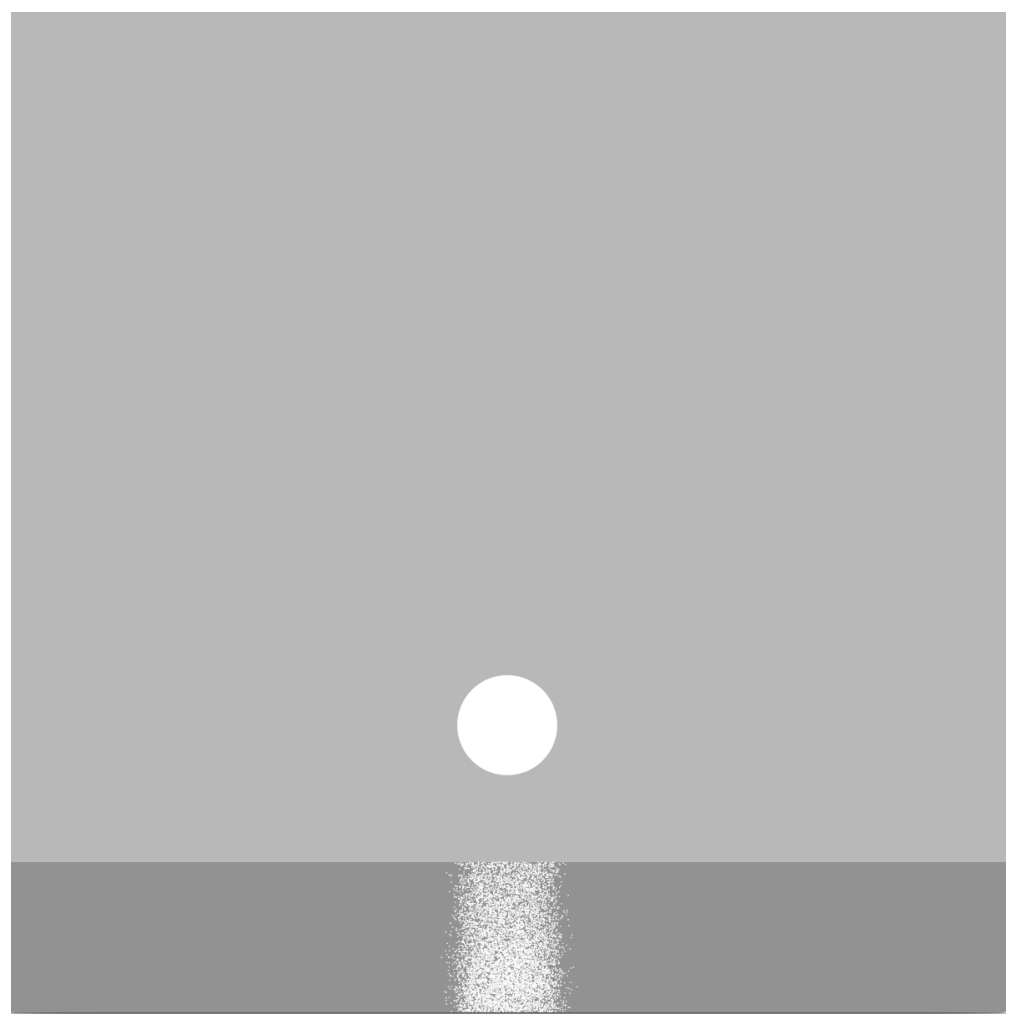

FIG. 5: Numerical simulation of the reflection at small angle of incidence of a light-emitting disc by a surface of randomly fluctuating orientation

To conclude we discussed a few ideas permitting to understand a quite common natural phenomenon, the blinking of the sea when the Sun is high and the formation of an apparent continuo stripe by reflection when the Sun is low. It seems $t$ us that besides its obvious aesthetical interest this phenomenon could ope the way to a better understanding of the reflection by a random surface with time dependent fluctuations. It is noticeable that this understanding follows from simple order of magnitude estimates. One could also wonder if it could be pertinent for other type of waves, like matter waves of quantum mechanics. 


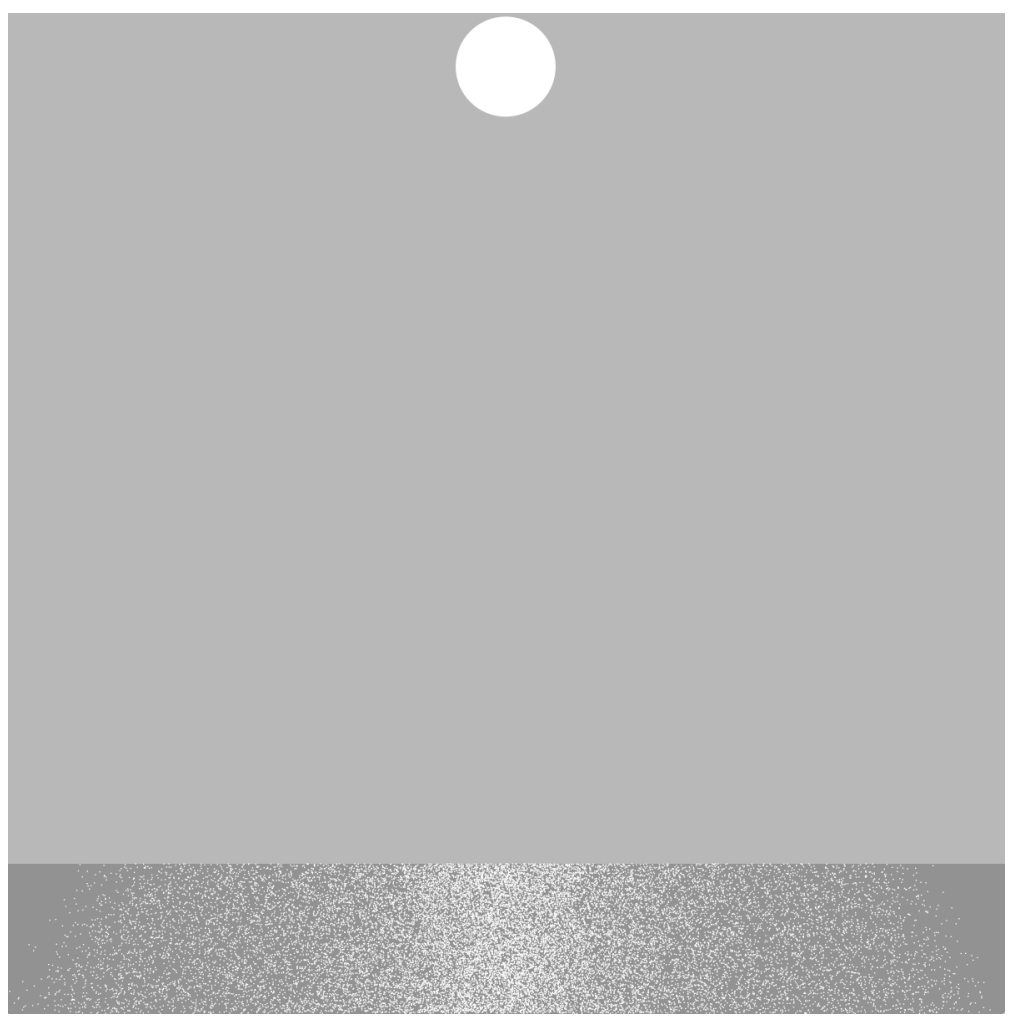

FIG. 6: Numerical simulation of the reflection at finite angle of incidence of a light-emitting disc by a surface of randomly fluctuating orientation

[1] C. Cox and W. Munk, "Statistics of the sea surface derived from sun glitter," J. Marine Res. 13, 198-227, (1954). C. Cox and W. Munk, "Measurement of the roughness of the sea surface from photographs of the Sun's glitter," J. Opt. Soc. Am. 44, 838-850, (1954).

[2] L.G. Aslamazov and A.A. Varlamov, "The Wonders of Physics" , World Scientific (2001), Singapore. 